\title{
A highly efficient channel sounding method based on cellular communications for high-speed railway scenarios
}

\author{
Liu Liu ${ }^{1 *}$, Cheng Tao ${ }^{1}$, Tao Zhou ${ }^{1}$, Youping Zhao ${ }^{1}$, Xuefeng Yin ${ }^{2}$ and Houjin Chen ${ }^{1}$
}

\begin{abstract}
An efficient channel sounding method using cellular communication systems is proposed for high-speed railway (HSR) propagation environments. This channel measurement technique can be used conveniently to characterize different HSR scenarios, which can significantly improve the measurement efficiency. Based on downlink signals of wideband code division multiple access (WCDMA) and the long term evolution (LTE), principles and methodologies of HSR channel sounding are presented. Using the WCDMA signal, a measurement campaign is conducted in real-world HSR scenarios and statistical characterizations are provided using a radio network analyzer. Due to the limits of the radio network analyzer, afterwards, a software defined radio (SDR)-based channel data recorder is developed allowing users to collect the signals from different wireless cellular systems. Especially, the estimation accuracies are validated in lab by the faded signals emitted from a vector signal generator. The results show that the channel data recorder provides a particularly good match to the configured fading channels. Therefore, this measurement method can be employed to investigate the HSR channel, and to establish the channel models under the various HSR scenarios.
\end{abstract}

\section{Introduction}

In recent years, broadband wireless access under highspeed railway (HSR) scenarios becomes a hot topic in industry and academia. With the development of HSR, more and more wireless data need to be transferred between the train and the ground [1]. On one hand, increasing railway controlling information consisting of security monitoring and maintenance needs to be transmitted from the train to the ground; on the other hand, mobile users are eager to have reliable multimedia services, independent of their locations or speeds. It is estimated that the wireless communication traffic could be as high as $65 \mathrm{Mbps}$ per high-speed train [2]. However, the existing communication systems cannot meet such requirements, therefore, how to offer high-quality wireless communication services to HSR has become an urgent issue for researchers, telecom operators and equipment manufacturers.

\footnotetext{
*Correspondence: liuliu@bjtu.edu.cn

${ }^{1}$ Institute of Broadband Wireless Mobile Communications, School of Electronic and Information Engineering, Beijing Jiaotong University, Beijing, China Full list of author information is available at the end of the article
}

A major prerequisite of any wideband digital radio system is a thorough knowledge of the propagation characteristics of the radio channel. Channel characterization is crucial in physical layer design, as it helps engineers design countermeasures to small-scale fading, such as diversity transmission/reception, error correction coding and interleaving, and equalization algorithms. Numerous wideband channel measurement campaigns have been performed to characterize urban radio channels for cellular mobile communication by researchers worldwide [3-5]. The measurement techniques can be categorized as: (1) Field strength measurements. In the actual deployment of a wireless communication system, the actual network planning optimization, capacity, blind spots and other studies are conducted according to the radio propagation environment. Accurate large-scale channel models (path loss and shadowing fading model) can make the network deployment and the network optimization reliable and efficient, so as to enhance the performance of wireless networks; (2) Measurements based on special excitation signals. This channel identification method employs a specific sequence possessing the Dirac-delta-like autocorrelation function, and correlates the received signal at the

\section{Springer}

C 2012 Liu et al: licensee Springer. This is an Open Access article distributed under the terms of the Creative Commons

Attribution License (http://creativecommons.org/licenses/by/2.0), which permits unrestricted use, distribution, and reproduction in any medium, provided the original work is properly cited. 
receiver with a copy of the signal identical to the transmitted one. Consequently, the channel impulse response (CIR) can be obtained.

Many of the previous studies on the HSR propagation measurement and characterization were conducted in recent years. The channel measurement using the GSM-R signal was taken on Zhengzhou to Xi'an (ZX) HSR. The experimental system is composed of a signal power recorder (Griffrin) combining with a GPS receiver. The received power amplitude was collected for the largescale fading parameterization [6-9]. In these published articles, the empirical path loss models under viaducts and U-shape Groove (USG) have been presented, whereas the features of small-scale fading are not included. In the pulse measurement, the characterization result of this measurement technique is mainly focused on a specified environment, which yields lower measurement efficiency. RUSK measurement campaign was conducted at $5 \mathrm{GHz}$ in Germany between Siegburg and Frankfurt [10,11]. An empirical path loss, shadow fading, $K$-factor, and other parameters related to the time delay spread as well as the angle spread were presented [11]. Propsound measurement was conducted at $2.5 \mathrm{GHz}$ in Taiwan [12], and the delay spread, maximum excess delay, temporal behavior of the Doppler power spectrum and Angles of Arrival/Departure are studied. Propsound measurement and characterization were carried out at $2.35 \mathrm{GHz}$ band by Beijing Jiaotong University [2]. A position-based wireless channel model for HSR viaduct scenarios was proposed. Based on the geometry-based stochastic model, the nonstationary MIMO Channel model was investigated [13].

In this article, our specific contributions to the HSR channel measurement and characterization are summarized as follows:

1) We provide an efficient channel sounding method using wireless cellular systems for HSR scenarios.

2) We specify the estimation methodology using downlink signal of wideband code division multiple access (WCDMA) and LTE as the excitation signal. The time delay parameters, Doppler properties and correlation matrices can be extracted.

3) Based on a radio network analyzer using WCDMA signal, a channel measurement is conducted in realworld HSR environments, and the time dispersive properties are provided under the typical terrains.

4) A prototype of the channel data recorder is developed to collect different cellular probing signals based on software defined radio (SDR) platform. Meanwhile, the key technologies are addressed. By employing WCDMA and LTE signals, we perform extensive measurements in lab for the calibrations using this recorder. The measured results show a good match.
The remainder of this article is organized as follows: In Section "Challenges in HSR channel characterization", we discuss the problems and motivations of the channel characterization in HSR radio environments. Section "Principles and methodology of HSR channel characterization based on cellular communication systems" addresses the principle and methodology of the channel sounding using WCDMA and LTE signals. In Section "Measurements on ZX and Beijing-Tianjin (BT) HSR", the measurement campaign conducted on HSRs is described and the time delay characteristics are provided. In Section "Wireless channel data recorder", the development of the channel data recorder and the calibrations in lab are discussed in more details. Section "Conclusions" draws the conclusions.

\section{Challenges in HSR channel characterization}

The knowledge of the wireless channel is vital to the optimal design and performance of any HSR wireless communication systems. Most of the previous literature on the channel measurement and characterization is focused on terrestrial cellular communication systems. Features of HSR propagation channels, such as time, frequency and spatial selectivity, differ from those of cellular communications channels. These differences originate from some specific features of the HSR propagation environments such as [2] (1) multiple scenarios; (2) line of sight (LOS) dominance; (3) large Doppler shift and rapid Doppler transition.

Compared with channel measurements for terrestrial cellular communication systems, it is very difficult to employ the commonly used channel sounding techniques in HSR environments for the reasons below:

(1) In Europe and China, communications between the train and railway administration control centers involving the train dispatching, marshaling shunting, maintenance repair operation and emergency in HSR systems are undertaken by GSM-R. In order to guarantee the security of this train control network, the wireless frequency bands are under surveillance by the railway administration. Any wireless signals are prohibited except the wireless communications running by the wireless operators, such as GSM, WCDMA, CDMA2000, etc. Therefore, the particular signal-based channel sounding campaign encounters a great deal of difficulties.

(2) In the static channel scenario, it is impossible to emulate the rapid change of the radio environment to extract the time-varying characterization of a given resolvable path component. Furthermore, the Doppler effect due to the high mobility has a considerable influence on the average fading rate of the discrete propagation wave. And the Doppler spread is resulting from different incoming waves of 
mobile vehicles and the scatters. For this reason, in order to extract the accurate Doppler property, achieving an adequate velocity is primary.

(3) Measurement efficiency is extremely low when the commonly used channel sounders are employed. If the train travels at a speed of $360 \mathrm{~km} / \mathrm{h}$ and the wireless coverage of a channel sounder is $1 \mathrm{~km}$, the recording time of the sounder approximates $20 \mathrm{~s}$. The obtained experimental data in this short period may be not adequate to extract the statistical property of the time-varying channels. Moreover, making a reservation for the measurement in a high-speed train, as well as the coordination of the rail-line and the dispatch, are exhausting. And it will take about a month for these preparatory work. Additionally, HSR encounters numerous typical terrains. It is time-consuming and costly to establish a complete and accurate propagation channel database using the universal channel sounders.

Considering the particular nature of the channel sounding for HSR scenarios, we explore a novel efficient channel sounding method using the signal from a wireless cellular system. This employed signal is treated as the excitation wave, and at the receiver side, the stochastic property can be extracted through post-processing.

\section{Principles and methodology of HSR channel characterization based on cellular communication systems}

Channel measurement and characterization with WCDMA signal

\section{Channel state estimation based on WCDMA signal}

WCDMA air interface employs the direct-sequence code division multiple technology and the frequency division duplex (FDD) mode. The chip rate is $3.84 \mathrm{Mcps}$ with the radio bandwidth of $5 \mathrm{MHz}$. This wideband signal possesses the large time bandwidth product, low crest factor and good correlation properties. These merits meet the requirements of a typical excitation signal [14].
The radio frame structure of WCDMA is shown in Figure 1 [15]. Frame structure has an overall length of $10 \mathrm{~ms}$, comprising 15 time-slots. The physical layer uses the Common Pilot Channel (CPICH) to gain an estimate of the downlink channel conditions. CPICH is a broadcast physical channel transmitting at a higher power than other dedicated channels. It does not take any service information. All mobiles in the cell can hear this signal. CPICH carries a pre-defined bit sequence 0000 ...... After QPSK modulation, it is spread with a constant spreading factor of all ones. Finally, this band-spread signal is scrambled by a cell specific scrambling code $S_{d l, n}$. Figure 2 shows the $\mathrm{CPICH}$ modulation procedure [16].

The typical WCDMA receiver can combine the signal contribution of a single propagation path using the Rake receiver. In our system, we aim to collect the channel information before the combination procedure. The employed channel sounder system is summarized in the functional block diagram of Figure 3. The received signal is down-converted to baseband and subsequently digitalized. After that, the time synchronization is done by synchronization channel $(\mathrm{SCH})$. Finally, channel conditions can be derived from the correlator using $\mathrm{CPICH}$.

Assume that time synchronization is achieved and the scrambling code is known. The sampled received signal, omitting the symbol-level details and replacing by the chip level, can be expressed as

$$
r(p)=\sum_{k=1}^{K} \sum_{l=1}^{L} c_{l}(p) a_{k}\left(p-\left[\frac{\tau_{l}}{T_{c}}\right]\right)+n_{k, l}(p),
$$

where $K$ is the number of channels in the physical layer within the frequency band, $L$ denotes the number of multipath components, $c_{l}(p)$ represents the discrete complex channel coefficient of $l$ th path, $a_{k}(p)$ stands for the discrete chip data of the $k$ th channel after scrambling and spreading, $\tau_{l}$ represents the delay of the $l$ th path, $T_{c}$ is the chip duration, $[x]$ means the rounding operation of $x$, and $n_{k, l}(p)$ denotes the additive white gaussian noise (AWGN) of the $l$ th multipath component in the $k$ th channel.

There are 15 time-slots in one CPICH frame, corresponding to 150 symbols. Each symbol is expanded into

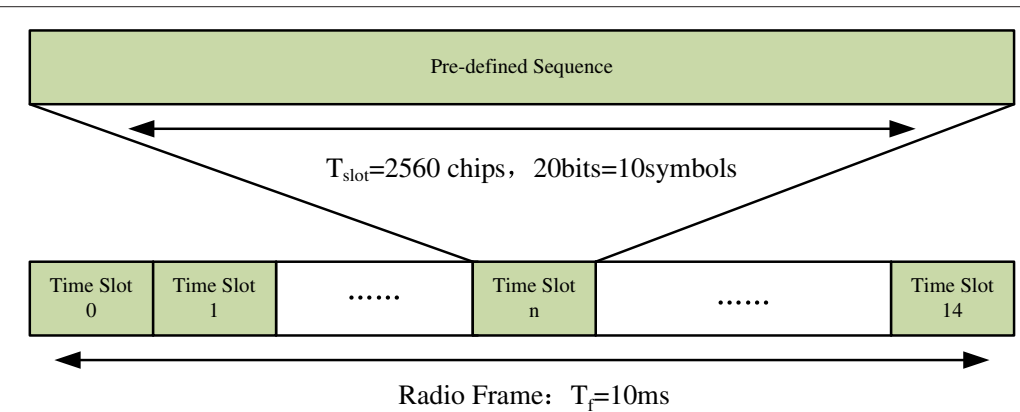

Figure 1 Frame structure of WCDMA. 


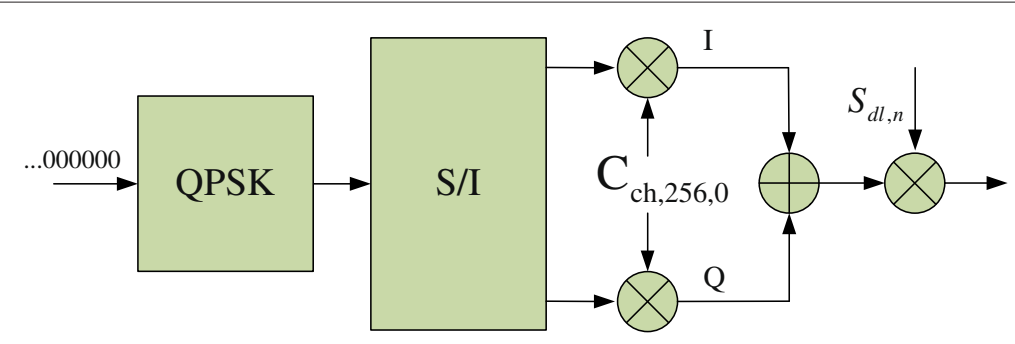

Figure 2 CPICH modulation procedure.

chip level by a fixed spreading factor of 256 with a spreading code of "all ones". All the symbols of the CPICH are $1+j$. Then (1) can be modified as

$$
\begin{aligned}
r(p)= & \sum_{l=1}^{L} c_{l}(p) s c\left(p-\left[\frac{\tau_{l}}{T_{c}}\right]\right) \\
& +\sum_{k=2}^{K} \sum_{l=1}^{L} c_{l}(p) a_{k}\left(p-\left[\frac{\tau_{l}}{T_{c}}\right]\right)+n_{k, l}(p),
\end{aligned}
$$

where the first term refers to $\mathrm{CPICH}$, the second term stands for the multiple-access interference (MAI) caused by other physical channels, and $s c(p)$ represents the scrambling code for a specified cell. Practical experiences reveal that both MAI and $n_{k, l}(p)$ terms can be considered as AWGN $w(p)$. In addition, there is some interferences in practice, which are caused by adjacent cells. This interferences are assumed to be negligible.

According to (2), the faded CPICH signal can be viewed as a sum of the multiple shifted copies of the scrambling code. After the correlation with the conjugation scrambling code, the estimated CIR can be expressed as

$$
\begin{aligned}
h(\tau)= & \frac{1}{N} \sum_{p=0}^{N-1} r(p+\tau) s c^{*}(p) \\
= & \frac{1}{N} \sum_{p=0}^{N-1} \sum_{l=1}^{L} c_{l}(p+\tau) s c\left(p+\tau-\left[\frac{\tau_{l}}{T_{c}}\right]\right) \cdot s c^{*}(p) \\
& +\frac{1}{N} w(p+\tau) s c^{*}(p)
\end{aligned}
$$

where $N$ is the size of the correlation window, and $\tau$ represents the correlation time index. For the balance between the correlation window and the snapshot rate (will be discussed in Section "The uncertainty principle in the time and frequency domains"), $N$ is chosen to be 256 . After some mathematic operations, the estimated CIR $h(\tau)$ becomes

$$
\begin{aligned}
h(\tau)= & \frac{1}{N} \sum_{p=0}^{N-1} c_{i}(p) \delta\left(\tau-\left[\frac{\tau_{i}}{T_{c}}\right]\right) \\
& +\frac{1}{N} \sum_{p=0}^{N-1} \sum_{l=1, l \neq i}^{L} c_{l}(p) s c\left(p+\tau-\left[\frac{\tau_{i}}{T_{c}}\right]\right) \\
& +\frac{1}{N} \sum_{p=0}^{N-1} w(p+\tau) s c(p),
\end{aligned}
$$

where the first term refers to the average of the channel coefficients, and the second term is inter-path interference (IPI) generated by other path components and does not align perfectly with the scrambling code phase. Since the scrambling code yields a peak as a $\delta$ function at the zero shift point, the IPI is usually weak when compared with the third term.

The uncertainty principle in the time and frequency domains In static channels, the excitation signal possessing the Dirac-delta-like autocorrelation is emitted periodically to

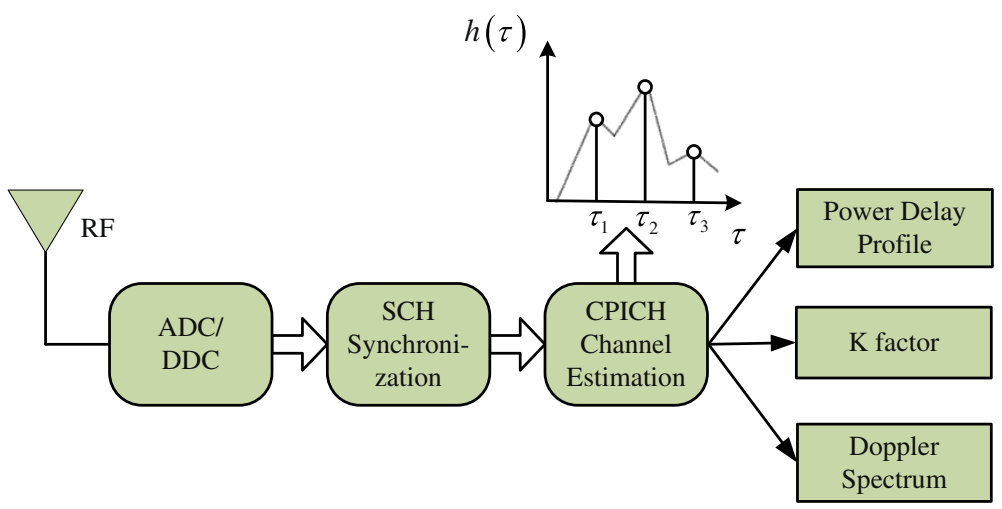

Figure 3 Functional block diagram of the channel sounder based on WCDMA. 
probe the channel. Therefore, the repetition period $T_{\text {rep }}$ of the sounding pulse must satisfy

$$
T_{\text {rep }} \geq \tau_{\max },
$$

where $\tau_{\max }$ is the maximum delay of the multipath component. Under this condition, the aliasing from echo waves can be avoided. On the other hand, in order to track instantaneous fading conditions, these repetitive signals need to be sent sufficiently frequently. $T_{\text {rep }}$ must be smaller than the time over which the channel changes. This notion can be formalized by establishing a sampling theorem in the time domain, so there is a minimum temporal sampling rate to identify a time-variant process with a band-limited Doppler spectrum. This temporal sampling frequency must be twice the maximum Doppler frequency $f_{\max }$ as

$$
f_{\text {rep }} \geq 2 f_{\text {max }} \text {. }
$$

Time-varying channels can be fundamentally unidentifiable because requirements for the design of sounding signals can become contradictory [14]. According to (5) and (6), it is intractable when we select the correlator window $N$ in (3). On one side, the time width $T_{W-\text { rep }}$ corresponding to $N$ has to be chosen larger than the maximum excess delay $\tau_{\max }$; On the other side, the repetition frequency $f_{\text {rep }}$ has to fulfill $T_{W-\text { rep }} \leq 1 / 2 f_{\max }$. The snapshot rate is inversely proportional to the correlator window width $N$. Consequently, if $N$ is made as small as possible, then the channel sampling rate increases. This will enhance the capability of tracking Doppler characteristics. However, this narrow window will increase the noise floor when we gather CIR, which will compress the dynamical range. It will make a significant effect on the resolvability of multipath components in lower SNR regions. In the data post-processing, therefore, the window width $N$ needs to be adjusted according to the measurement scenario.

Channel measurement and characterization with LTE signal Long term evolution (LTE) of the 3rd Generation Partnership Project. Orthogonal frequency division multiplexing (OFDM) and multiple-input and multiple-output (MIMO) are the key technologies in LTE system. Like WCDMA, the OFDM signal also can be employed as the excitation signal for channel sounding [17]. The downlink signal of LTE is competent for channel sounding for HSR due to its broader bandwidth of $20 \mathrm{MHz}$.

\section{Channel state estimation based on LTE signal}

The basic frame has an overall length of $10 \mathrm{~ms}$. It is further subdivided into ten $1 \mathrm{~ms}$ subframes, each of which is split into two $0.5 \mathrm{~ms}$ slots (as shown in Figure 4 [18]). Each slot comprises seven individual OFDM symbols in the case of the normal cyclic prefix length. The LTE system has been conceived to work under high-mobility assumptions. In the time-frequency plane, a uniform reference symbol (RS) grid with a diamond shape is used. The required spacing in time between the RSs can be obtained by considering the maximum Doppler spread (highest speed) to be supported, which for LTE corresponds to $500 \mathrm{~km} / \mathrm{h}$. In the frequency direction there is one RS every six subcarriers in each OFDM symbol including RS, but they are staggered so that within each Resource Block (RB) there is one RS every three subcarriers corresponding to $45 \mathrm{KHz}$. Thus it allows the feasibility of the channel estimation of the expected frequency domain.

The LTE downlink has been specifically designed to work with multiple transmit antennas, and the specific RS is orthogonal with each other antenna. For simplicity, the single antenna system is described in this section. MIMO measurement system can be easily generated with this method. Here we address the channel estimation problem over one OFDMA symbol (all the subcarriers being $\mathrm{RSs}$ ) to exploit the frequency domain characteristics. The channel transfer function (CTF) can be estimated using a maximum likelihood approach in the frequency domain by de-correlating the constant modulus RS. Then, the CTF of the overall subcarriers can be obtained by interpolation methods [19].

Assuming that there are $N$ subcarriers in an OFDM symbol, $X[\mathrm{~m}]$ is the complex-valued training data on the $m$ th subcarrier, which is actually known at the receiver. After $N$-point IFFT, the transmitted signal $x(n)$ can be expressed as

$$
x(n)=\frac{1}{N} \sum_{m=0}^{N-1} X[m] \exp \left(j 2 \pi \frac{m n}{N}\right) \quad 0 \leq n \leq N-1 .
$$

Then the convolved output between the excitation signal $x(n)$ and the CIR in time domain can be written as

$y(n)=h(n, l) * x(n)+w(n)=\sum_{l=0}^{L-1} h(n, l) x(n-l)+w(n)$.

First, the channel is assumed as time-invariant, namely $h(n, l)=h(l)$. Then at the receiver, after the synchronization, CP removal and FFT operation, the received signal in frequency domain is

$$
\begin{aligned}
Y[m] & =\left(\sum_{l=0}^{L-1} h(l) e^{-j 2 \pi l k / N}\right) X[m]+W[m] \\
& =H[m] X[m]+W[m],
\end{aligned}
$$




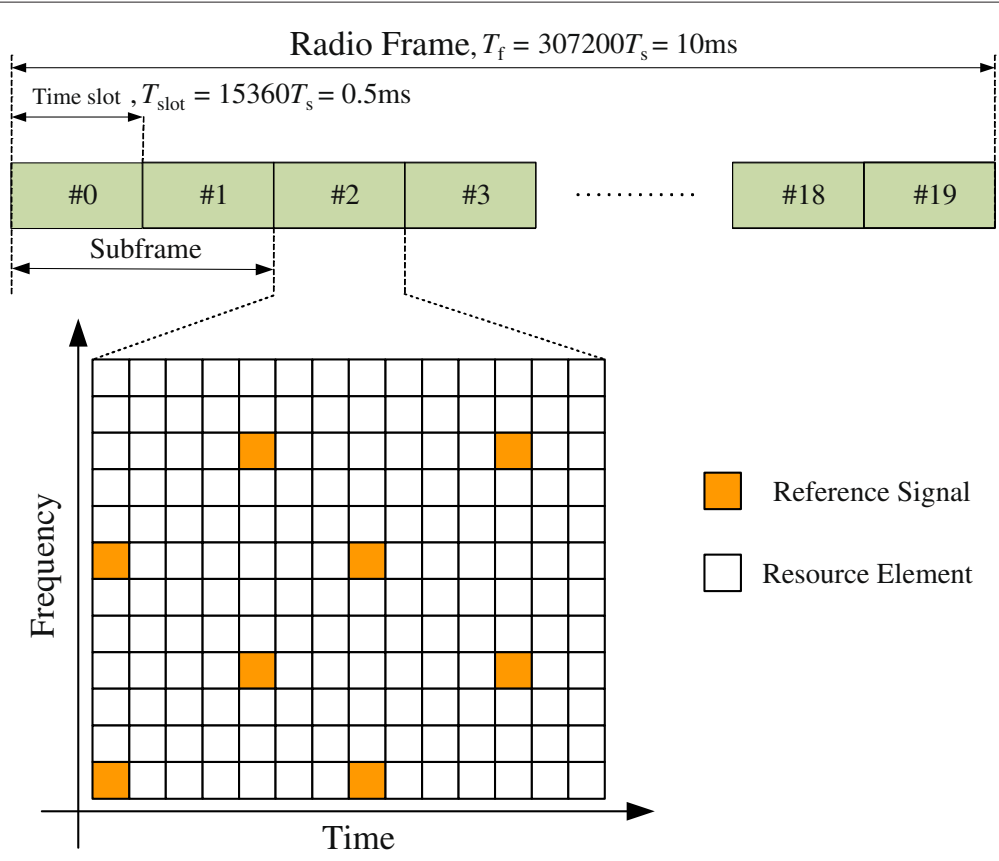

Figure 4 Frame format of LTE and the pilot grid.

where $H[m]$ and $W[m]$ refer to the frequency response and AWGN at $m$ th subcarrier. Then a simple correlation processing in frequency domain is performed as

$$
\tilde{H}[m]=\frac{Y[m] X^{*}[m]}{|X[m]|^{2}}=H[m]+W^{\prime}[m] .
$$

Finally, an estimate of the impulse response $\tilde{h}(l)$ can then be obtained from (10) via the Fourier series as

$$
\tilde{h}(l)=\sum_{n=0}^{N-1} \tilde{H}[m] e^{j 2 \pi l m / N} .
$$

\section{The spatial correlation measurement with LTE signal}

A dominant LOS component occurs in viaduct scenarios of HSR [2], whereas the performance of MIMO systems depends strongly on the underlying propagation conditions. An important parameter of the MIMO channel is the spatial correlation. The antenna separation also has a significant impact on the spatial correlation. The LTE downlink has been specifically designed to work with multiple transmit antennas, and the cell-specific RS can be used for the estimation of the spatial correlation. The RS grid of two antenna ports is shown in Figure 5 [18]. In

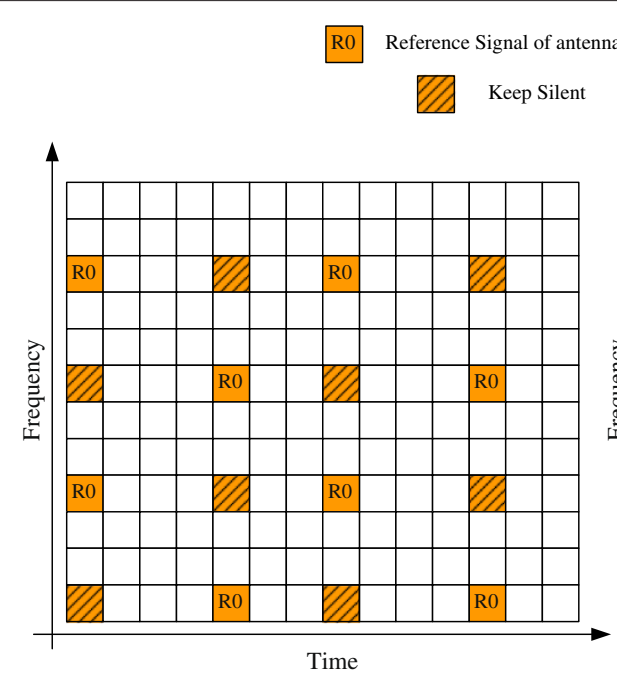

R1 Reference Signal of antenna 1

$\square$ Resource Element

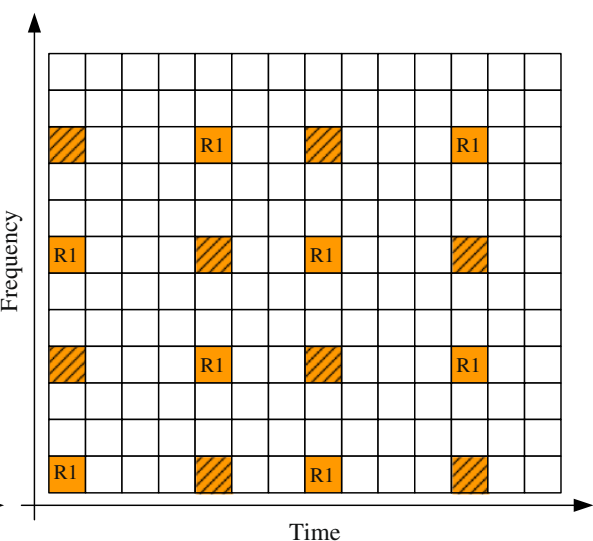

Figure 5 Pilot grid structure of two-antenna transmitter system. 
order to keep the orthogonality of the RSs, superposed positions in the different antennas are not used for transmission, namely keep silent. Using the channel estimation as described in the previous section, the CIR of each antenna also can be derived.

Among the possible spatial correlation models, Kronecker model is used [20]. This kind of channel is based on the assumption that scatters around the transmitter are uncorrelated with respect to those around the receiver. Furthermore, it is assumed that the fading correlation properties of all the resolvable multipaths have the same correlation characteristics. In the $2 \times 2 \mathrm{MIMO}$ case, the evolved Node B (eNB) and User Equipment (UE) correlation matrices are defined, respectively, as follows

$$
\mathbf{R}_{\mathrm{eNB}}=\left(\begin{array}{ll}
1 & a \\
a^{*} & 1
\end{array}\right), \quad \mathbf{R}_{\mathrm{UE}}=\left(\begin{array}{ll}
1 & b \\
b^{*} & 1
\end{array}\right)
$$

and the spatial normalized correlation of $4 \times 4$ MIMO case is defined as

$$
\begin{aligned}
\mathbf{R}_{\mathrm{spat}}=\mathbf{R}_{\mathrm{eNB}} \otimes \mathbf{R}_{\mathrm{UE}}= & {\left[\begin{array}{lllr}
1 & a^{1 / 9} & a^{4 / 9} & a \\
a^{1 / 9 *} & 1 & a^{1 / 9} & a^{4 / 9} \\
a^{4 / 9 *} & a^{1 / 9 *} & 1 & a^{1 / 9} \\
a^{*} & a^{4 / 9 *} & a^{1 / 9 *} & 1
\end{array}\right] } \\
\otimes & {\left[\begin{array}{llll}
1 & b^{1 / 9} & b^{4 / 9} & b \\
b^{1 / 9 *} & 1 & b^{1 / 9} & b^{4 / 9} \\
b^{4 / 9 *} & b^{1 / 9 *} & 1 & b^{1 / 9} \\
b^{*} & b^{4 / 9 *} & b^{1 / 9 *} & 1
\end{array}\right], }
\end{aligned}
$$

where $\otimes$ is the Kronecker product.
The parameters $a$ and $b$ in (12) define the spatial correlation between the antennas at the UE and eNB. Then the correlation coefficient is given by

$$
\rho_{p q}=\frac{E\left[h_{p} h_{q}^{*}\right]-E\left[h_{p}\right] E\left[h_{q}^{a s t}\right]}{\sqrt{\left(E\left[\left|h_{p}\right|^{2}\right]-\left|E\left[h_{p}\right]\right|^{2}\right)\left(E\left[\left|h_{q}\right|^{2}\right]-\left|E\left[h_{q}\right]\right|^{2}\right)}}
$$

where $h_{p}$ and $h_{q}$ denote the CIR of the $p$ receiving antenna and the $q$ transmitting antenna, respectively. ()* represents the conjunction operation.

\section{Measurements on ZX and Beijing-Tianjin (BT) HSR Descriptions of the measurement campaign}

The R\&S TSMQ Radio Network Analyzer can be employed for network analysis and optimization, and it can extract the resolvable multipath component corresponding to a specific cell [21]. The number of paths, path location (in the delay domain), and path attenuation can be derived by the supporting software. All of the behaviors, such as synchronization and channel estimation are accomplished by TSMQ. Practical testing experiences reveal that the maximum resolvable time delay is $20 \mu \mathrm{s}$ and the minimum resolvable path power is $-20 \mathrm{~dB}$ when employing TSMQ.

In our measurement, both Direct Coverage and High Speed and Mobile Cell (proposed in [2]) are considered. The measurement configuration is plotted in Figure 6. It is noted that the measurement on ZX HSR follows the Direct Coverage scheme in which the receiver antenna is positioned inside the carriage, whereas the antenna is positioned on the top of the train roof in BT HSR measurement. Besides, single-input and single-output is considered [22].

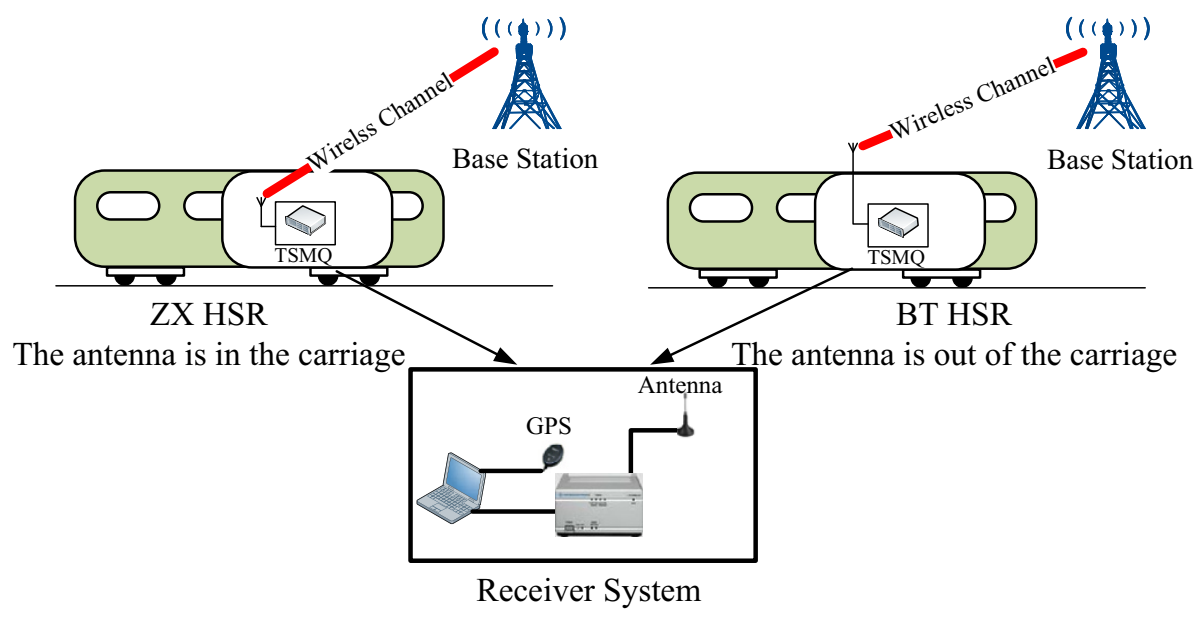

Figure 6 Configuration of the measurement campaigns on ZX and BT HSRs. 


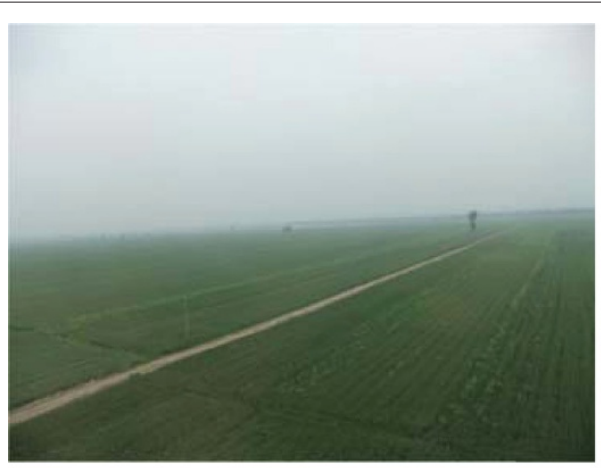

(a)

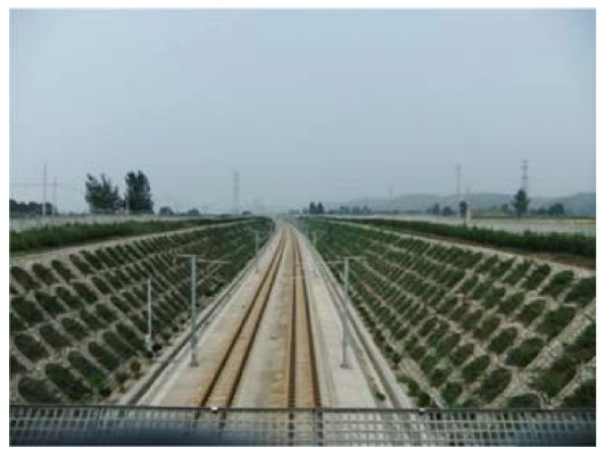

(c)

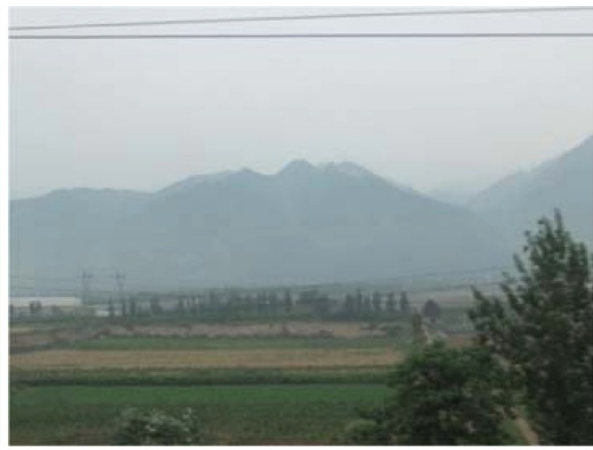

(b)

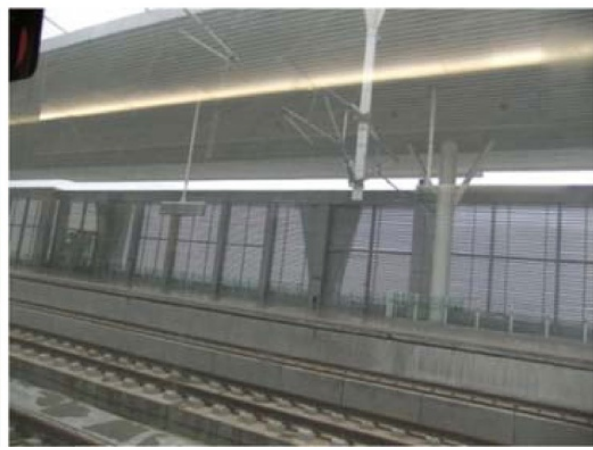

(d)

Figure 7 Typical HSR scenarios.

Figure 7 shows several typical scenarios such as the rural area, hilly terrain, groove area and a railway station. In the rural area, there are open terrains with agricultural fields. No specular scatters and reflectors are observed around the rail. In the Hilly Terrain, the measurement track is chosen with a plain environment on one side and a mountain at a distance of $800 \mathrm{~m}$ on the other side. In these two cases, the WCDMA base stations are situated at a distance of $30 \mathrm{~m}$ from the rail. USG is a specific construction of rail with double slope sidewalls resulting in a semi-enclosed propagation environment. The base station antenna is mounted on top of one slope wall with a separation of $10 \mathrm{~m}$. The measured groove is selected near the North of HuaShan Railway Station, with a depth of about $5 \mathrm{~m}$ and a length of $1000 \mathrm{~m}$. The Railway Station scenario is distinct from the regular terrain. The newly built station is made of metal materials that yield more complex additional reflected and scattered components. The selected platform is approximately $100-\mathrm{m}$-long, $45-\mathrm{m}$-wide and 30 - $\mathrm{m}$-high. BS antenna is located at the entrance with height of $35 \mathrm{~m}$.

BT measurement is conducted in the No. 0 high speed integrated inspection train (NHSIIT) with a speed of approximately $240 \mathrm{~km} / \mathrm{h}$ using the train-mounted antenna. NHSIIT is used to check the train system admin- istrated by the Ministry of Railways of China. The BT environment is composed of the viaduct and roadbed, with small roadside bushes and agricultural fields in between.

\section{Channel parameters estimation on ZX HSR}

One of the primary channel statistics of interest for digital communications applications is the delay spread, which quantifies the range of delays between the first received impulse and the last, and describes the distribution of the amplitude. The probability density function (PDF) of root mean square (rms) delay spread and number of paths are shown as the basic channel parameters [23].

Figure 8 depicts the rms delay spread for the four scenarios. The Plain and Hilly Terrain show the similar distribution and yield the smallest values of rms delay spread. This means that the single path dominates in these two environments with more than $84 \%$ of rms being zero. The largest rms percentage, $0 \mu \mathrm{s}$, encountered for USG is approximately $60 \%$, and for the Railway Station case, it accounts less than $10 \%$.

Figure 9 shows the PDF of the number of resolvable multipath components. The cases of one propagation wave occupy the dominant positions in the Plain or 

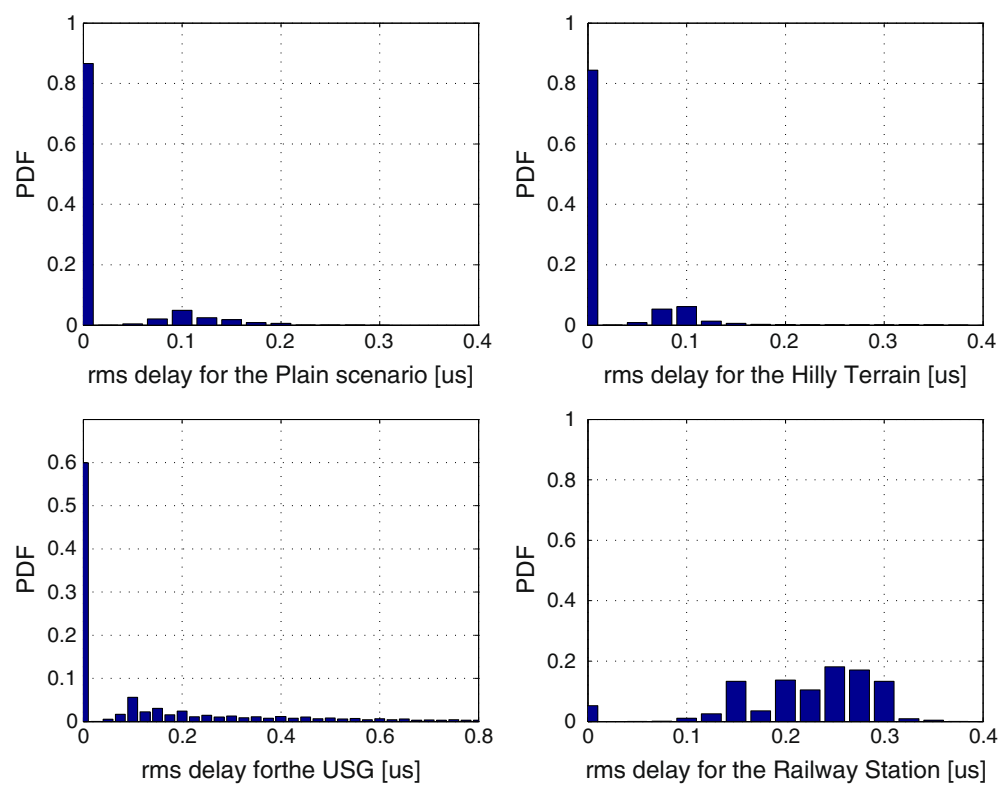

Figure 8 rms delay spread in different scenarios.

Hilly Terrain scenarios, with probabilities of 87 and $86 \%$, respectively. The results are seen to be in good agreement with conclusions of rms delay spread investigated above. Under the Railway Station condition, significant multipath components are more obvious.

On the basis of the preceding discussion, we conclude that the Plain and Hilly Terrain scenarios have similar time dispersive behaviors because the mountain in Hilly Terrain is relatively far from the rail, the reflected wave is too weak to be detected by TSMQ after the penetration loss of the carriage. Whereas, Railway Station and USG show significantly stronger multipath effects. In Railway Station scenario, radio waves will undergo the reflection many times, which induced from isolated components from reflections of strong stationary scatterers, e.g., the wall, pillar and roof of the platform. In USG environment diffuse reflections, caused by weak static scatterers such as cement slope sides, arise frequently.

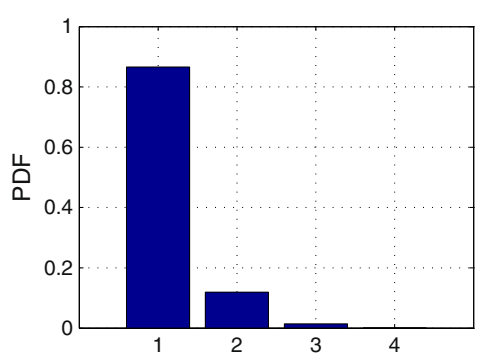

Number of paths for the Plain scenario

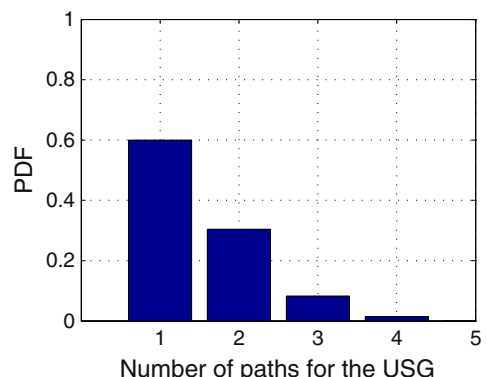

Number of paths for the USG

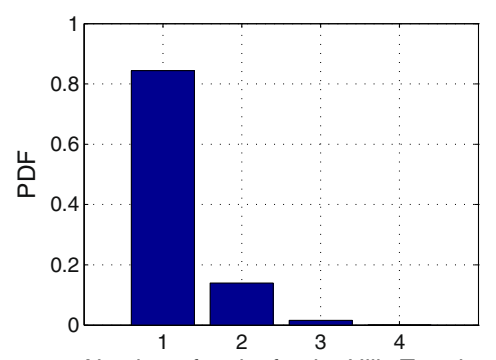

Number of paths for the Hilly Terrain

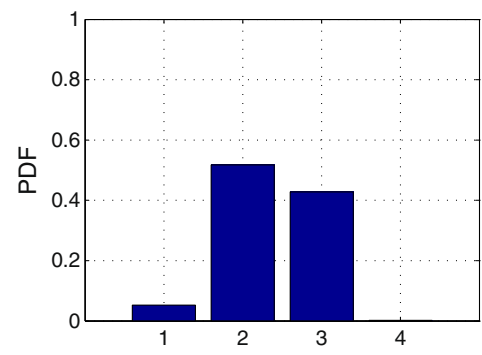

Number of paths for the Railway Station

Figure 9 Number of paths in different scenarios. 
In nearly all of the treatments, the channel is modeled as a tap delay line (TDL) filter [24]. Based on the collected data, using maximum likelihood criterion, we parameterize the number of taps, the relative time delay and the average path gain of TDL models for the four scenarios in Table 1.

\section{Channel parameters estimation on BT HSR}

Table 2 lists the channel delay parameters in the two different plains on BT HSR, A and B area. It exhibits explicitly in most cases, there is only one propagation wave, i.e., rms delay spread is zero in the snapshots with the probabilities of 98 and $91 \%$, respectively. The reason is that the BT line runs over the plain area, and the train-mounted antenna on the roof is applied. In this scheme, the radiated signal directly arrives the receiver antenna without any effect from the penetration fading, reflection and scattering inside the train. This provides a benign environment for the mobile radio provision.

Especially for BT HSR, the leading ray (LOS component) is investigated. The received signal envelop has a Ricean distribution with the corresponding probability density function given by [25]

$$
P(r)=\frac{r}{\sigma^{2}} \cdot \exp \left(-\frac{r^{2}+s^{2}}{2 \sigma^{2}}\right) \cdot I_{0}\left(-\frac{r \cdot s}{\sigma^{2}}\right)
$$

where $I_{0}()$ is the 0th-order modified Bessel function of the first kind, $r$ denotes the envelope of the received signal, $\sigma^{2}$ represents the variance of the diffuse components, and

Table 1 Channels for the typical terrains

\begin{tabular}{lccc}
\hline Scenario & Tap number & $\begin{array}{c}\text { Relative time } \\
\text { delay }[\boldsymbol{\mu s}]\end{array}$ & $\begin{array}{c}\text { Average path } \\
\text { gain [dB] }\end{array}$ \\
\hline Plain & 1 & 0 & 0 \\
& 3 & 0.3 & -12.9 \\
& 1 & 0.6 & -22.2 \\
Hilly Terrain & 2 & 0 & 0 \\
& 3 & 0.3 & -7.6 \\
& 1 & 0.6 & -22 \\
USG & 2 & 0 & 0 \\
& 3 & 0.3 & -7.3 \\
& 4 & 0.9 & -22.9 \\
Railway Station & 1 & 2.1 & -24 \\
& 2 & 0 & -8.2 \\
\hline & 3 & 0.3 & 0 \\
\hline
\end{tabular}

Table 2 Delay spread characteristics on BT HSR

\begin{tabular}{lcc}
\hline Scenarios & $\mathbf{r m s}=\mathbf{0}$ & Maximum number of multipath components \\
\hline Terrain A & $98 \%$ & 2 \\
Terrain B & $91 \%$ & 2 \\
\hline
\end{tabular}

$s^{2}$ is the power of the direct LOS path component. Any moment of the Ricean distribution can be given as

$$
\begin{aligned}
\mu_{n} & =E\left[r^{n}\right] \\
& =2\left(\sigma^{2}\right)^{\frac{n}{2}} \exp (-k) \Gamma\left(1+\frac{n}{2}\right) F_{1}\left(1+\frac{n}{2} ; 1 ; k\right),
\end{aligned}
$$

where $\Gamma(\cdot)$ is the gamma function, and $F_{1}(\cdot)$ is the confluent hypergeometric function. Then the estimated $K$ factor can be obtained as [25]

$$
\hat{K_{2,4}}=\frac{-2 \hat{\mu}_{2}^{2}+\hat{\mu}_{4}-\hat{\mu}_{2} \sqrt{\hat{\mu}_{2}^{2}-\hat{\mu}_{4}}}{\hat{\mu}_{2}^{2}-\hat{\mu}_{4}},
$$

where $\mu_{2}$ and $\mu_{4}$ are the even moments.

Table 3 provides the $K$-factor results that collected in the environments. Scenario A and B are the viaduct models, exhibiting a stronger LOS, with the $K$ factor of 4.8 and $5.2 \mathrm{~dB}$, respectively. Actually, the $K$-factor is around $5 \mathrm{~dB}$ in most instances in the entire measurement campaign on BT HSR.

\section{Wireless channel data recorder System description}

As previously mentioned, it is extremely convenient to employ the TSMQ for channel sounding based on the radio signals. In addition to its portability, all of the estimation behaviors, such as cell search, synchronization, noise floor selection and path search are accomplished by the equipment. Unfortunately, this device has proprietary algorithms and techniques. Users cannot improve the algorithms to enhance the accuracy of the channel parameter characterization. In the case of TSMQ, the phases of the multipath components cannot be provided. For this reason, we are unable to extract the Doppler characteristic which is significant for HSR channels. Therefore, a SDRbased channel data recorder is developed. The functional block diagram is shown in Figure 10. In this recorder, intermediate frequency (IF) sampling is selected to minimize the DC biased components and reduce the complexity inherent in hardware for in-phase and quadraturephase demodulation. Then, the digital down conversion

Table $3 K$-Factor on BT HSR

\begin{tabular}{lcc}
\hline Scenarios & A & B \\
\hline K-Factor & 4.8 & 5.2 \\
\hline
\end{tabular}




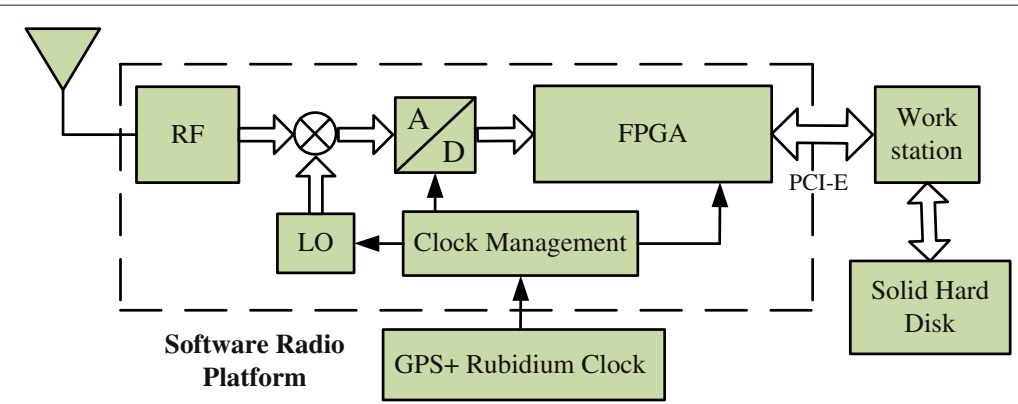

Figure 10 Functional block diagram of the channel data record.

Table 4 Parameters of wireless channel data recorder

\begin{tabular}{llll}
\hline Parameter & Value & Parameter & Value \\
\hline Bandwidth & $100 \mathrm{MHz}$ & A/D Bitwidth & 14 bits \\
RF & $2.1 \mathrm{GHz} \sim 2.6 \mathrm{GHz}$ & Interface & PCl-E Bus \\
Local clock reference & GPS + Rubidium clock & Storage medium & Solid hard disk $512 \mathrm{~GB}$ \\
Max sampling rate & $200 \mathrm{MSPS}$ & Power & $12 \mathrm{~V}$ \\
\hline
\end{tabular}

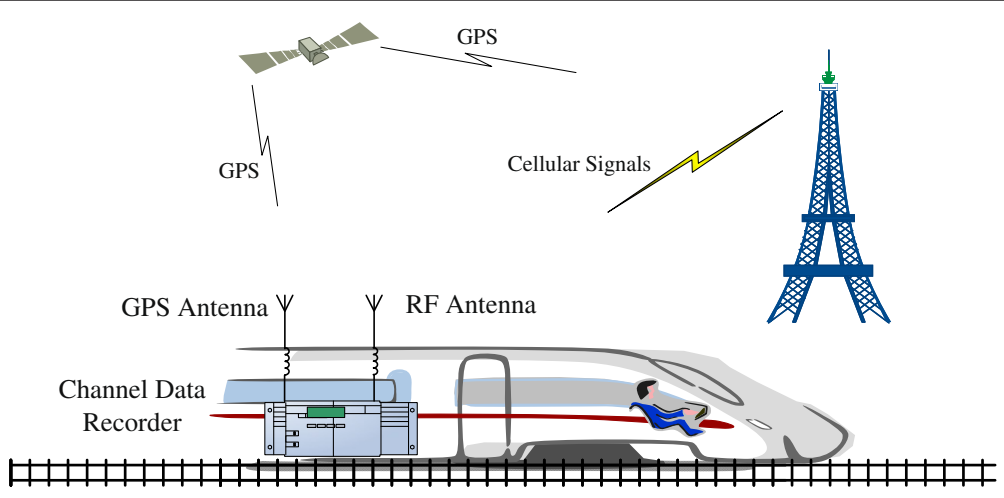

Figure 11 GPS-based time synchronization method for the channel data recorder on HSR.

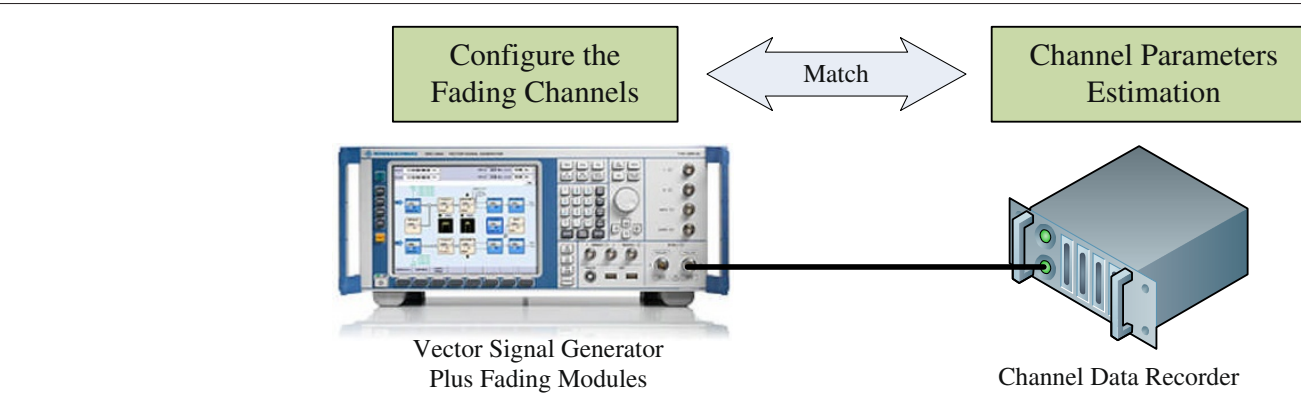

Figure 12 Principle for the calibration in lab. 
Table 5 Emulation channels for channel data recorder using WCMDA

\begin{tabular}{llccc}
\hline Channel & Tap number & $\begin{array}{c}\text { Relative } \\
\text { delay }[\boldsymbol{\mu s}]\end{array}$ & $\begin{array}{c}\text { Average path } \\
\text { gain [dB] }\end{array}$ & $\begin{array}{c}\text { Doppler } \\
\text { spectrum }\end{array}$ \\
\hline Rayleigh/ & 1 & 0 & 0 & CLASS/RICE \\
Rice & 2 & 0.52 & -3 & CLASS \\
& 3 & 1.04 & -6 & CLASS \\
& 4 & 1.56 & -9 & CLASS \\
\hline
\end{tabular}

(DDC) and filtering are done in FPGA. The digital baseband signals are transferred to a workstation via the PCI-E bus. The real sampled signals are stored in a solid hard disk with a data transfer rate up to $600 \mathrm{MB} / \mathrm{s}$. The parameters are listed in Table 4.

According to the functional block, the benefits of this recorder are described as follows:

(1) We can configure different estimation software according to different excitation signals at different frequencies to extract the channel properties owing to the wideband radio frequency $(R F)$ interface.

(2) In our recorder, the signals with multiple frequencies, multiple spread codes, and various communication standard signals can be stored into the hard disk simultaneously. The extraction procedure of the target signal can be accomplished by softwares off-line. This enhances the measurement efficiency significantly. In the real measurement employing TSMQ, in order to achieve the higher sampling rate, we have to configure the parameters of a specific cell in real-time, including the central frequency, spreading code, etc. The measurement task using TSMQ is intensive.

The synchronization between the transmitter and the receiver is a key technology for the wireless channel sounding. The accurate synchronization in frequency and time domains is crucial to the correct sampling the incoming signal, simultaneous switching of multiple antennas, and extraction of Doppler characteristics. In the outdoor environment, the transceiver may be separated by distances up to dozens of kilometers. The Global Positioning System (GPS) offers a way of establishing common time and frequency references. This reference signal can be employed for our channel data recorder in large range measurements as shown in Figure 11. The channel data recorder and the cellular base station receive the GPS signal for the synchronization simultaneously. This timing signal can, in turn, be used to synchronize the local oscillator and sample clocks at the base station and the recorder. This clock link is able to provide an accurate universal timing signal, typically $10^{-12}$ to $10^{-14} \mathrm{~s}$. The GPS synchronization technique can be used anywhere where there is access to the GPS network, which is justifiably outdoors with a good LOS to the sky.

\section{Laboratory calibration}

The channel identification using the channel data recorder needs to be verified in lab (as shown in Figure 12). We employ the vector signal generator SMU200A by R\&S. This equipment has integrated a digital fading simulator which can emulate typical time-varying multipath fading channels. The main procedure is described as follows:

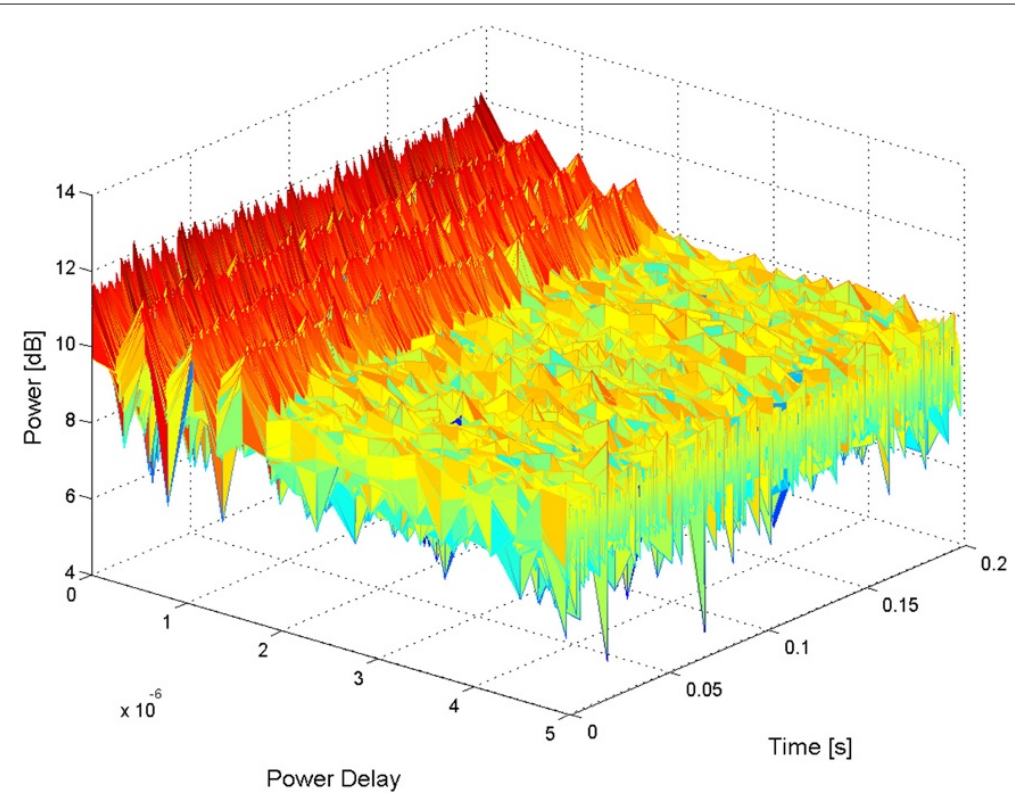

Figure 13 Time-varying PDP based on WCDMA signal. 


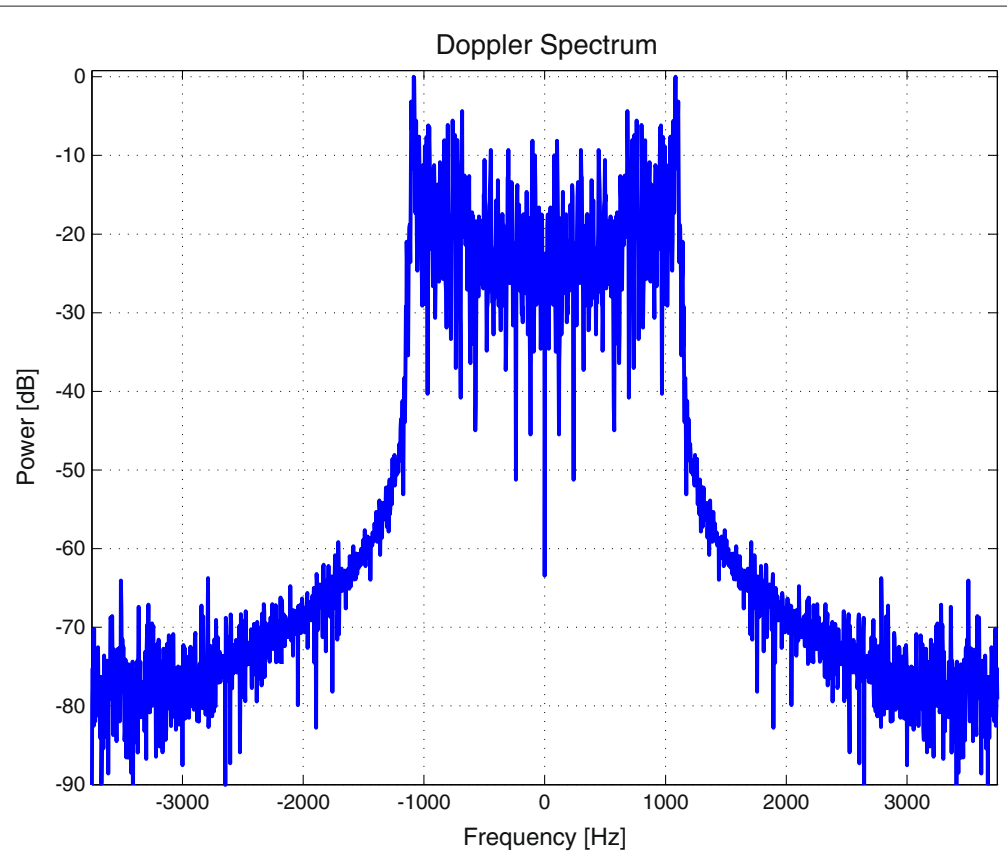

Figure 14 JAKES Doppler spectrum based on WCDMA signal.

(1) the pure cellular signals are set in the generator, such as WCDMA, LTE; (2) the typical fading channel parameter are configured, such as COST 207 and ITU channel model; (3) connect the RF cables, and then the faded signal is fed to the recorder; (4) sample the received signals, extract the channel parameters and make comparisons between the set model and derived results.

\section{Verified results based on WCDMA signal}

In WCDMA calibration, $\mathrm{SCH}, \mathrm{CPICH}$ and dedicated physical channel are used. And the transmit power is $-10 \mathrm{dBm}$. Table 5 lists the parameters of the evaluation models in which both LOS and Non-line of sight (NLOS) scenarios are considered. The mobile velocity is $500 \mathrm{~km} / \mathrm{h}$. In order to prevent interferences from the operating cellular system, and the central frequency is set to $2.4 \mathrm{GHz}$.

Figure 13 shows the time-varying power delay profile (PDP) with the duration of $0.2 \mathrm{~s}$ corresponding to 20 radio frames. The PDP can be represented as [26]

$$
P_{P D P}(i, k \Delta \tau)=\frac{1}{W} \sum_{n=i W}^{(i+1) W-1}\left|h\left(n t_{\mathrm{rep}}, l \Delta \tau\right)\right|^{2}
$$

where $t_{\text {rep }}$ is the snapshot repetition period, $\Delta \tau$ denotes the time delay resolution, $\mathrm{W}$ is the window size for averaging. It can be found that the measured path delay and relative power of PDP present a good match with the channel parameters listed in Table 5.
Doppler characteristic also can be derived from continuous snapshots. The estimated Doppler spectrum is given as

$$
\operatorname{DPP}(r \Delta v, k \Delta \tau)=\mathrm{fft}[\operatorname{corr}(h(k \Delta \tau))],
$$

where $\mathrm{ftt}$ is the fast Fourier transformation, corr is the correlation operation. For the LOS case, $\Delta \tau=0$. As can be seen in Figure 14, the measured power spectrum is consistent with classical $U$-shape spectrum and the maximum Doppler frequency offset approximates $1110 \mathrm{~Hz}$.

Compared with the urban environment, LOS component dominates in HSR scenarios. $K$-factor can be extracted from the CIRs. Table 6 shows the measured results of $K$-factor with a decreasing trend, from 15 to $-5 \mathrm{~dB}$. It is obvious that our results provide better approximations in the stronger LOS scenarios.

The RICE Doppler spectrum is comprised of a LOS component and numerous scattering echo waves. According to (19), we plot the Doppler spectrum of the first arriving propagation signal as in Figure 15. It is observed that one spectrum line locates at $560 \mathrm{~Hz}$ corresponding to the incidence angle of $60^{\circ}$.

\section{Table 6 Performance of $K$-factor estimation}

\begin{tabular}{lccccc}
\hline Set value $\boldsymbol{K}$ [dB] & $\mathbf{1 5}$ & $\mathbf{1 0}$ & $\mathbf{5}$ & $\mathbf{0}$ & $\mathbf{- 5}$ \\
\hline Estimated value & 14.96 & 9.96 & 5.04 & 0.07 & -3.95 \\
$\begin{array}{l}\text { Relative error of } \\
\text { estimated value }\end{array}$ & $0.8 \%$ & $0.1 \%$ & $1 \%$ & $1.7 \%$ & $2.1 \%$ \\
\hline
\end{tabular}




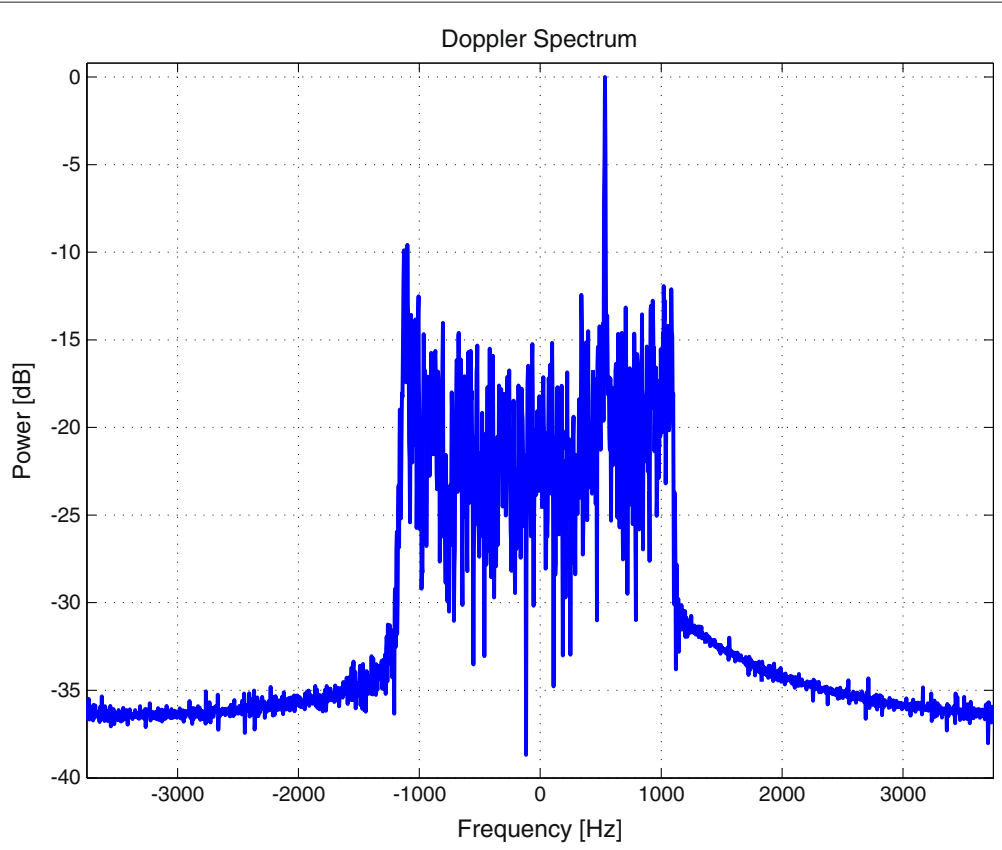

Figure 15 RICE Doppler spectrum based on WCDMA signal.

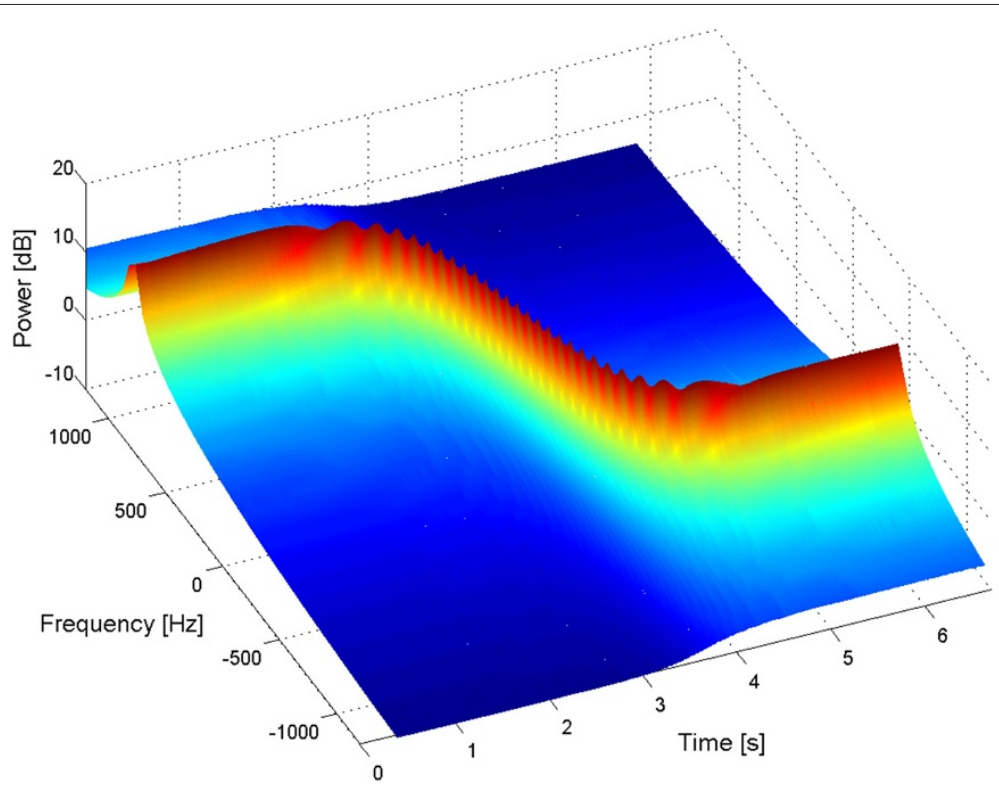

Figure 16 Rapid Doppler transition in HSR scenario based on WCDMA signal.

Table 7 Parameters of wireless channel data recorder

\begin{tabular}{llll}
\hline Parameter & Value & Parameter & Value \\
\hline Frequency & $2.6 \mathrm{GHz}$ & Duplex mode & FDD \\
Bandwidth & $5 \mathrm{MHz}$ & Min resolvable time $T_{M}$ & $0.22 \mu \mathrm{S}$ \\
Channel delay & {$[0,2,3,5,8,11] \times T_{M}$} & Relative power & {$[0-1-9-10-15-20] \mathrm{dB}$} \\
Velocity & {$[0,120,250,350] \mathrm{km} / \mathrm{h}$} & Doppler spectrum & CLASS \\
\hline
\end{tabular}


Table 8 Estimation errors [dB] of time delay characteristics using LTE signal

\begin{tabular}{lcccc}
\hline Item & $\mathbf{0 ~} \mathbf{~ m} / \mathbf{h}$ & $\mathbf{1 2 0} \mathbf{~ k m} / \mathbf{h}$ & $\mathbf{2 5 0} \mathbf{~ k m} / \mathbf{h}$ & $\mathbf{3 5 0} \mathbf{~ k m} / \mathbf{h}$ \\
\hline $\mathrm{SNR}=0 \mathrm{~dB}$ & 0.1814 & 0.1956 & 0.1983 & 0.1856 \\
$\mathrm{SNR}=10 \mathrm{~dB}$ & 0.0633 & 0.0624 & 0.0747 & 0.0696 \\
$\mathrm{SNR}=20 \mathrm{~dB}$ & 0.0557 & 0.0608 & 0.0495 & 0.0630 \\
\hline
\end{tabular}

Table 9 Spatial fading correlation calibration using LTE signal

\begin{tabular}{lccc}
\hline Item & $\begin{array}{c}\text { Low } \\
\text { correlation } \\
\boldsymbol{\alpha}=\mathbf{0}\end{array}$ & $\begin{array}{c}\text { Medium } \\
\text { correlation } \\
\boldsymbol{\alpha = 0 . 3}\end{array}$ & $\begin{array}{c}\text { High } \\
\text { correlation } \\
\boldsymbol{\alpha}=\mathbf{0 . 9}\end{array}$ \\
\hline $\mathrm{SNR}=-10 \mathrm{~dB}$ & 0.0034 & 0.2990 & 0.7945 \\
$\mathrm{SNR}=0 \mathrm{~dB}$ & 0.0014 & 0.3007 & 0.8951 \\
$\mathrm{SNR}=10 \mathrm{~dB}$ & -0.0098 & 0.3009 & 0.8994 \\
$\mathrm{SNR}=20 \mathrm{~dB}$ & -0.0051 & 0.3042 & 0.8997 \\
\hline
\end{tabular}

In 3GPP TS36.104 [27], the high-speed train conditions for the test of the baseband performance are proposed in open space and tunnel scenarios for multi-antennas. Here, the eNB-Railway distance is $50 \mathrm{~m}$ and the velocity is $350 \mathrm{~km} / \mathrm{h}$. The instantaneous Doppler power spectrum is plotted in Figure 16. In this figure the Doppler spectrum line sweeps from the maximum to the minimum value as the train passes through the entire wireless coverage. It obviously shows that the Doppler frequency offset changes with the variation from $+780 \mathrm{~Hz}$ to $-780 \mathrm{~Hz}$.

\section{Verified results based on LTE signal}

The sounding parameters for the LTE system are listed in Table 7. In Table 8, we provide the estimation errors against the different velocities under varying SNR conditions. The results evidently represent that SNR has a significant impact on the estimation accuracy. But the velocity factor does not degrade the estimation performance.
Similarly, the Doppler spectrum can be directly extracted from the continuous snapshots of CIRs, which we will not go further.

MIMO conformance test is also verified. Our experimental calibration works on the narrowband condition. And for simplicity, first, we employ two transmitting antennas and one receiving antenna. Three spatial correlation values with low, medium and high are considered and the performance results are listed in Table 9 [27]. It indicates that in the low and medium cases, the estimated accuracies perform approximately independent of SNR and the relative errors are negligible. In contrast, high correlation cases are more susceptible to the noise level which encounters lager variances. This is due to the fact that in lower SNR cases, the extracted results are intensely disturbed by the random noise which will yield the reduction of the correlation.

In addition, four transmit and four receive antennas are also considered. The low, medium and high values, at the transmitter and receiver, are considered in case of $\mathrm{SNR}=10 \mathrm{~dB}$. Figure 17 demonstrates explicitly that the calibrated results match well with the configured parameters.

\section{Conclusions}

This article focuses on the development of a novel and efficient channel sounding method for HSR environments, which directly exploits the radio signals of existing cellular systems. We investigate the principle and methodology of the use of WCDMA and LTE as the excitation signals. Afterwards, HSR radio channel measurements are conducted with TSMQ, which provide time-dispersive characteristics of typical HSR scenarios. Due to the shortcomings of the commercial measurement devices, a SDRbased channel data recorder is developed featuring convenient signal sampling structure and competent data processing. The experimental results are calibrated in lab compared with the configured fading models, which
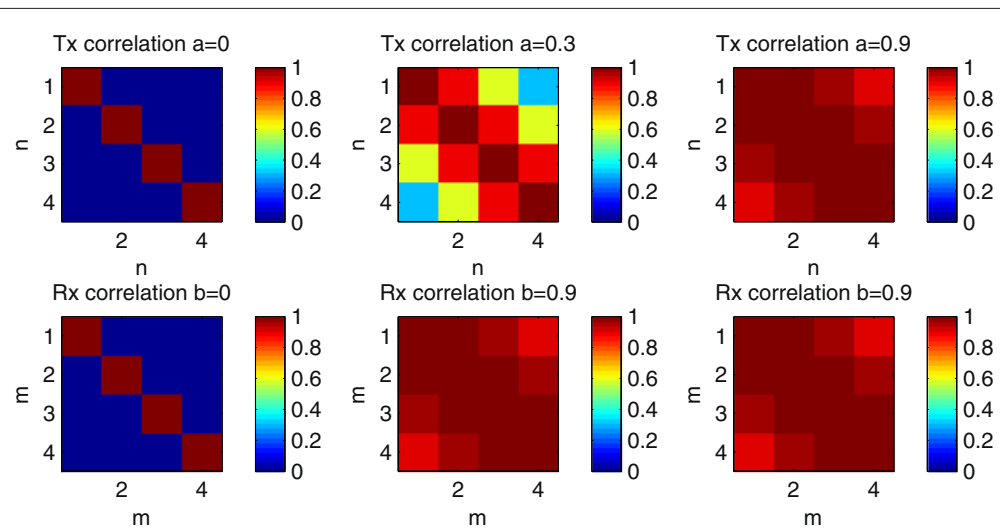

Figure 17 Correlation matrices of four transmit and four receive antennas system. 
shows a good match. By virtue of the high efficiency of this novel method, employing this channel data recorder, our setup system should be a useful tool to characterize HSR propagation channels.

\section{Competing interests}

The authors declare that they have no competing interests.

\section{Acknowledgements}

The research was supported in part by the NSFC project under grant Nos. 61032002 and 61102050, the National Science and Technology Major Project under grant 2011ZX03004-006, the Beijing Natural Science Foundation project under grant No. 4122061, and the Fundamental Research Funds for the Central Universities under grant No.2012YJS005.

\section{Author details}

${ }^{1}$ Institute of Broadband Wireless Mobile Communications, School of Electronic and Information Engineering, Beijing Jiaotong University, Beijing, China.

${ }^{2}$ School of Electronics and Information Engineering, Tongji University, Shanghai, China.

Received: 14 February 2012 Accepted: 21 June 2012

Published: 3 October 2012

\section{References}

1. Y Zhou, Z Pan, J Hu, J Shi, X Mo, in Proc. 20th Annual Wireless and Optical Communications Conf. (WOCC). Broadband wireless communications on high speed trains, (Newark, New Jersey, USA, 2011), pp. 1-6

2. L Liu, C Tao, J Qiu, H Chen, L Yu, W Dong, Y Yuan, Position-based modeling for wireless channel on high-speed railway under a viaduct at 2.35 GHz, IEEE J. Sel. Area. Commun. (J-SAC) 30(4), 834-845 (April 2012)

3. M Failli, Digital Land Mobile Radio Communications COST 207, Final Report. Tech. rep, (Commission of the European Communities, Luxembourg, 1989)

4. E Damosso, Digital Mobile Radio Towards Future Generation Systems COST 231 Final Report. Tech. rep., Office for Official Publications of the European Communities (THE COMMISSION OF THE EUROPEAN COMUNITIES, Luxembourg, 1996)

5. LM Correia, Wireless Flexible Personalised Communications: COST 259, European Co-operation in Mobile Radio Research, (Wiley, Chichester, UK, 2001)

6. L Gao, Z Zhong, B Ai, L Xiong, in Proc. 5th Int Communications and Networking in China (CHINACOM) ICST Conf. Estimation of the ricean $\mathrm{K}$ factor in the high speed railway scenarios, (Beijing, China, 2010), pp. 1-5

7. H Wei, Z Zhong, K Guan, B Ai, in Proc. 5th Int. Communications and Networking in China (CHINACOM) ICST Conf. Path loss models in viaduct and plain scenarios of the high-speed railway, (2010), pp. 1-5

8. R He, Z Zhong, B Ai, J Ding, An empirical path loss model fading analysis for high-speed railway viaduct scenarios, IEEE Antenn. Wirel. Pr. 10 808-812 (2011)

9. $\mathrm{R} \mathrm{He}$, Z Zhong, B Ai, J Ding, Propagation measurements analysis for high-speed railway cutting scenario, Electron. Lett. 47(21), 1167-1168 (2011)

10. MEDAV: RUSK Measurement Campaigns Overview, MEDAV GmbH (Germany, 2008)

11. K Pekka, WINNER II Channel Models Part II Radio Channel Measurement and Analysis Results (2007)

12. R Parviainen, K Pekka, in European Cooperation in the Field of Scientific and Technical Research, EURO-COST 2100. Results of high speed train channel measurements, (2008), pp. 1-6

13. A Ghazal, CX Wang, H Haas, M Beach, X Lu, D Yuan, X Ge, in Proc. IEEE VTC12-Spring Conf., A non-stationary, MIMO channel model for high-speed train communication systems, (Yokohama, Japan, May 2012), pp. 1-5

14. A Molisch, Wireless Communications, 2nd edn (John Wiley \& Sons Ltd, The Atrium, Southern Gate, Chichester, West Sussex, PO19 8SQ, United Kingdom, 2011)

15. Physical Channels and Mapping of Transport Channels onto Physical Channels FDD(Release 6), 3GPP TS 25.211, V6.10.0 (2009)

16. Spreading and Modulation FDD (Release 7), 3GPP TS 25.213 V7.7.0 (2009)
17. F Hlawatsch, G Matz, Wireless Communications Over Rapidly Time-Varying Channels. (Academic Press, MA, 2011)

18. Evolved Universal Terrestrial Radio Access (E-UTRA); Physical Channels and Modulation (Release 9), 3GPP TS 36.211, V9.1.0 (2010)

19. SG Kang, YM Ha, EK Joo, A comparative investigation on channel estimation algorithms for OFDM in mobile communications, IEEE Trans. Broadcast. 49(2), 142-149 (2003)

20. J Kermoal, L Schumacher, K Pedersen, P Mogensen, F Frederiksen, A stochastic, MIMO radio channel model with experimental validation, IEEE J. Sel. Area. Commun. 20(6), 1211-1226 (2002)

21. Radio network analyzer operating manual, ROHDE \& SCHWARZ, (Germany, 2010)

22. J Qiu, C Tao, L Liu, Z Tan, in Proc. IEEE VTC12-Spring Conf. Broadband channel measurement for the high-speed railway based on WCDMA (2012), pp. 1-5

23. TS Rappaport, Wireless Communications: Principles and Practice (Prentice Hall, Upper Saddle River, NJ, 1999)

24. MC Jeruchim, KS Shanmugam, P Balaban, Simulation of Communication Systems: Modeling, Methodology, and Techniques (Kluwer Academic Publishers, New York, 2000)

25. GL Stuber, Principles of Mobile Communication, 2nd edn (Kluwer Academic Publishers, USA, 2001)

26. A Paier, J Karedal, N Czink, C Dumard, T Zemen, F Tufvesson, A Molisch, C Mecklenbrauker, Characterization of Vehicle-to-Vehicle Radio Channels from Measurements at 5.2GHz, Wirel. Personal Commun. 50, 19-32 (2009)

27. Evolved Universal Terrestrial Radio Access (E-UTRA); Base Station BS Radio Transmission and Reception (Release 10), 3GPP TS 36.104, V10.5.0 (2011)

doi:10.1186/1687-1499-2012-307

Cite this article as: Liu et al.: A highly efficient channel sounding method based on cellular communications for high-speed railway scenarios. EURASIP Journal on Wireless Communications and Networking 2012 2012:307.

\section{Submit your manuscript to a SpringerOpen ${ }^{\mathcal{O}}$ journal and benefit from:}

- Convenient online submission

- Rigorous peer review

- Immediate publication on acceptance

- Open access: articles freely available online

- High visibility within the field

- Retaining the copyright to your article

Submit your next manuscript at $\boldsymbol{\wedge}$ springeropen.com 